\title{
Gravity revealed as electromagnetic force
}

\author{
Larry E. Thiele \\ L.E. Thiele Consulting, Green Bay, WI 54313 USA \\ Corresponding author: Larry E. Thiele (e-mail: ethiele@ netnet.net, website: www.LEThieleConsulting.com). \\ Funded entirely by Larry E. Thiele and L.E. Thiele Consulting.
}

May 27, 2021

\begin{abstract}
The earth is fundamentally protons, electrons, and neutrons. The force of gravity on earth could simply be a phenomenon of those elements. Lacking is any analysis demonstrating how electric charge forces of protons and electrons, both repulsive and attractive, can give rise to a gravitational force so much weaker and only attractive. Here application of Coulomb's law of electric charges shows the force of gravity derives from the basic proton-electron charge force. Separation of electrons from protons within any atom results in infinitesimal force imbalances, either repulsive or attractive, with every external proton-electron pair. When such force imbalances are accumulated using a Monte Carlo probability simulation for all charge pair in a large mass like the earth, repulsive forces are shown to never entirely cancel attractive forces and a weak net attractive force always remains. Coulomb's law yields the same force between earth and an object at its surface as Newton's law of gravity, confirming that gravity is an electromagnetic force and not a unique force of its own. This research is a mathematical analysis, an application of basic scientific principles much like the computer modeling of a complex engineered system. It has been done with no need for new theories, new speculation, abstract reasoning, nor abstract mathematics.
\end{abstract}

\section{INTRODUCTION}

Gravity on earth could just be an attraction between charge elements within the earth and charge elements within objects at and beyond the surface. Until the present time no analysis has been performed attempting to prove this relation. Even though Newton presented his law of gravity in 1686 describing how the force of gravity between two masses is proportional to the product of the mass quantities and inversely proportional to the square of the separation distance, he had no answer for what causes the force of gravity [1]. Gravity remained much of a mystery until 1915 when Einstein published his paper on general relativity [2]. Even in that paper Einstein did not specifically address gravity as a force nor identify what causes the force of gravity on earth. In a subsequent book published by Einstein in 1916 in which he attempted to simplify the presentation of relativity to the general public [3], he did not mention a source of the force of gravity. In spite of this, there also is no evidence in these publications that Einstein denied gravity being an actual force. Einstein continued a relentless search during the latter part of his life to find a link between electricity and gravity, but that search yielded no answers to what causes the force of gravity [4]. Richard Feynman in his lectures on gravitation in 1963 [5] said attempts to unify gravitation with electrodynamics had been primarily unsuccessful mathematical games not worthy of further discussion. He did however admit that gravity might be the consequence of something we already know but have not calculated correctly, perhaps having to do with charge. Other attempts have been made to link gravity with electromagnetics [6] but none have successfully identified electric charge as the source of the force of gravity. 


\section{APPLICATION OF COULOMB'S LAW}

Coulomb's law of electric charges [7][8] states the electric force between two charges is proportional to the product of the charge quantities divided by the square of their separation distance:

$$
F=\frac{k q_{1} q_{2}}{R^{2}}
$$

Here $F$ is the force, $q_{1}$ and $q_{2}$ are the charge quantities, $R$ is the separation distance, and $k$ is the proportionality constant. Coulomb's law, dating back to 1785 , is the unquestioned trusted foundation for all electric theory and the underlying basis for all modern electric, electronic, and communication technology. Coulomb's law does not preclude the existence of a force between any two charges regardless of their separation distance, location in the universe, and intervening charge or mass. Electric charge forces of protons and electrons do not abruptly cease at atomic boundaries, crystal boundaries, nor boundaries of higher matter. Charge forces are always present between charge elements within any atom, between charge elements among adjacent atoms, and between charge elements among distant atoms.

Coulomb's law identifies three fundamentals: (1) quantity of charge entities or particles that are influenced or experience a force, i.e., protons and electrons, (2) distance separating the particles, and (3) force between the separated particles. Charge therefore is a consequence of these three fundamentals and not some unique influence or property in addition to these three. "Charged" means a condition whereby an excess quantity of one type charge element exists over the other type charge element. In Coulomb's law, as it is applied to the atoms comprising a higher mass like the earth, charge is a representation of a quantity proportional to the count of protons and electrons. Charge influence of a proton and electron is nothing other than the Coulomb force between the proton and electron.

The analysis herein recognizes that the properties and actions of an atom are not known with absolute certainty. The analysis does not depend on knowledge of exactly where an electron is located at any time nor how and in what direction it changes locations. Therefore an electron's location and movement within an atom are considered to be random, except an electron does not occupy at any instant of time the exact same location as any proton, an electron occupies at any instant of time a single specific location rather than being in multiple places at once, any electron over time can occupy multiple different locations randomly distributed within a space surrounding the protons, and an electron's separation distance from the protons exhibits an average value over time.

Given that Coulomb's law describes a force between any two charges, then every proton and electron charge within every atom in the entire earth should experience a force with every other proton and electron within the earth and every proton and electron within every mass object at the earth's surface and beyond. For a large mass like the earth and an object at its surface, the total Coulomb force is the sum of individual Coulomb forces for all charge elements comprising the masses. Therefore, any imbalance between repulsive and attractive forces results in a net nonzero Coulomb force between the earth and the surface object. Therein lies the basis for the gravitational force being a net force imbalance between electric charges.

Several critical outcomes are necessary to show equivalence with Newton's gravity law for large masses: (1) a nonzero imbalance in charge forces can exist between local charge elements (protons and electrons) and remote charge elements despite zero net charge at both locations, (2) these force imbalances between local and remote on earth are so much weaker (on the order of $10^{36}$ times) than the charge force between any single proton and electron separated by the same distance, (3) the accumulation of all such force imbalances for a large mass is only attractive in any direction, and (4) Newton's gravity law can be derived. 


\section{INTERPRETING ZERO NET CHARGE}

In electric theory electroneutrality or zero net charge means count of protons equals count of electrons within a given space. Within an increment of mass the count of electrons normally equals the count of protons and since the quantity of charge assigned to each proton and electron is equal in magnitude but opposite in sign, the total net charge of the mass calculated as the sum of individual charge quantities is zero. Despite this zero net charge, a force still exists between each local charge element versus any remote charge element according to Coulomb's law. There is no imbalance in charge quantity to consider for any electroneutral mass, but any imbalance in separation distances between charges gives rise to an imbalance in charge forces between local and remote.

According to fundamental electric theory, when two adjacent mass objects become charged where one acquires an excess positive charge and the other an excess negative charge, the unequal distribution of charge quantity between the objects makes the objects no longer electroneutral. In that case an electric field and voltage can be detected to exist between the objects along with a force. When the objects then discharge, the charge distribution across each object returns to equal between positive and negative and the objects are again electroneutral. No detectable electric field or voltage is then present and therefore no detectable electrical force is said to exist between the objects. This is how electric theory associates zero external Coulomb forces with zero net charge. This can lead to a mistaken belief that electroneutrality, or zero net proton-electron charge, can never produce any net external charge forces. That will be shown here to be not true.

For a large mass like the earth the gravitational force at any location is basically constant over time. Charge forces of the earth's protons and electrons are however constantly changing with respect to time as the electrons move within their atoms and separation distances with remote protons and electrons constantly change. Coulomb's law is not a function of time. It describes a force between charges at a single instant of time, an instantaneous force. It is the instantaneous values of charge forces that need to be accumulated as an average value over time and over the entire earth in order to compare to the gravitational force. The analysis is therefore a search for the time-averaged sum of Coulomb forces associated with randomly distributed electrons.

For a single proton-electron pair within a local atom with respect to a single proton-electron pair within a remote atom at a distance considerably beyond atomic bonding distances, total Coulomb force between local and remote is the vector sum of four individual forces: local proton versus remote proton, local proton versus remote electron, local electron versus remote proton, and local electron versus remote electron. Two have positive signs and two have negative signs. Despite net charge of both the local pair and remote pair being zero, it is incorrect to assume net force between local and remote is zero.

First consider just the local proton-electron pair versus the remote proton. Because the electron occupies space around its proton and is not ever at the exact same location as the proton, the local proton and electron are normally at different separation distances with respect to the remote proton. At most instances of time these two local-remote forces are then not equal in magnitude and their sum gives a net nonzero value. Separation distance and forces vary with time. Therefore, local-remote forces must be calculated over time to determine if the time-averaged net force is also nonzero.

Let both the local proton's location and the remote proton's location be fixed along the $x$-axis. At any instant let $F_{\text {total }}$ be the sum of the $x$-axis components of force between local (position 1) and remote (position 2), $p$ designate the protons, $e$ designate the local electron, $R$ the proton-proton separation distance, $r$ the local proton-electron separation distance, and $x_{e}$ the $x$-axis component of the local proton- 
electron separation distance. In this simple case when the local electron is located on the $x$-axis at $+x_{e}$ between the two protons:

$$
\begin{gathered}
F_{\text {total }}=F_{p 1 p 2}+\left(F_{\text {el } p 2}\right)_{x-a x i s} \\
F_{\text {total }}=\frac{k q_{p 1} q_{p 2}}{R^{2}}+\frac{k\left(-q_{e 1}\right)\left(q_{p 2}\right)}{\left(R-x_{e}\right)^{2}}
\end{gathered}
$$

A negative sign represents an attractive force and a positive sign a repulsive force. A time-averaged value of forces can be calculated as the local electron occupies different locations around its proton. For every position with a positive value of $x_{e}$ the electron likely experiences a position on the opposite side of its proton with a negative value of $x_{e}$. While the electron is between the two protons, the net force $F_{\text {total }}$ is attractive, and while the electron is outside the two protons, the net force $F_{\text {total }}$ is repulsive. Therefore, as the electron alternates at these positions on both sides of its proton on the $x$-axis, a net force wave is created varying between attractive and repulsive. However, due to the inverse of the square of the separation distance, the magnitude of the attractive force at $+x_{e}$ is greater than the magnitude of the repulsive force at $-X_{e}$, i.e., the time-averaged sum of these forces with the remote proton is nonzero and attractive, as determined by:

$$
\frac{1}{\left(R+x_{e}\right)^{2}}<\frac{1}{\left(R-x_{e}\right)^{2}}
$$

This example shows that a nonzero time-averaged charge force imbalance can exist between local and remote charge elements despite zero net charge at both locations. Also, three factors have already been identified which contribute to the substantial weakening of the total local-remote force imbalance compared to proton-electron forces within an atom. First there is the decrease in force due to the inverse square of the separation distance between local and remote per Coulomb's law. Second, the imbalance in local-remote force produced by the local proton and electron reflects an infinitesimally small difference in local-toremote separation due to the infinitesimally small separation distance between the local proton and electron. Third, the time averaging of forces as the electron occupies positions on opposite sides of its proton results in summing positive force imbalances with negative force imbalances over time producing a net average value further reduced in magnitude.

Next consider that a proton-electron pair also exists at the remote location instead of just a single proton. The total force between local and remote is then the sum of four forces:

$$
F_{\text {total }}=F_{p 1 p 2}+F_{e 1 p 2}+F_{p 1 e 2}+F_{e 1 e 2}
$$

The two protons produce a mutual repelling force, the two electrons produce another mutual repelling force, and there are two mutual attracting forces, one between the local electron and remote proton and a second between the remote electron and local proton. Based on the discussion above, each proton-electron attracting force can have a net time-averaged value greater than the time-averaged value of the protonproton repelling force. What remains is to determine the net time-averaged value of the electron-electron repelling force and whether the two attracting forces dominate. The exact location of either electron around its proton at any time cannot be determined so no exact calculation of local-remote forces can therefore be made. However, a valid approximation can be made by means of a Monte Carlo [9] probability simulation. In addition to the random positions associated with two pair of proton-electron charges, there is the simultaneous effect of random positions occurring for all proton-electron pair within the same atom and within adjacent atoms for any small increment of mass. Some of these force imbalances are net attractive 
and some are net repulsive. The combined average of all random force imbalances is a valid approximation of the net force on the mass increment.

\section{METHOD SUMMARY}

The essence of the Monte Carlo simulation is to determine if there is a consistent, identifiable force pattern that results from the separation between local and remote electrons randomly located within the spaces around their protons. The electrons change locations with time and their separation between local and remote therefore varies with time. The simulation needs to determine if the resulting time-varying Coulomb forces produce a consistent pattern in average value of the time-variations. By simulating a large sample of random electron locations, a distribution of the most probable values of instantaneous force can be calculated and from that an average value of force.

A computerized Monte Carlo simulation is performed by randomly positioning electrons in a spherical space around their protons and calculating the net imbalance for the four force components at these positions. Because an electron maintains a time-averaged separation from its proton, a spherical surface is selected as the electron space to examine. By repeating a large sample of calculations, each with new random electron positions, the most expected time-averaged force imbalance can be determined. The exact time period over which averaging of forces occurs is not relevant as long as it is great enough to provide assurance of a high probability that any location on the spherical surface can be occupied by the electron in the time period. Sequence of occurrence is also not relevant with time averaging. The average force over time is calculated for different relative positions of the local and remote electrons, which can be both inside the two protons, both outside the two protons, or one inside and the other outside the protons. When combined with the constant proton-proton repulsive force, the result determined is how often the sums of repulsive forces are dominated by the two proton-electron attractive forces.

\section{MONTE CARLO SIMULATION EQUATIONS}

The unique nature of this force calculation involves taking the difference between two forces with magnitudes differing by a small amount. Consider for the quantity $R-x_{e}$ that $R=6 \times 10^{6}$ at a separation distance equal to the radius of the earth and $x_{e}=1 \times 10^{-10}$ for the proton-electron separation distance within an atom. A straight forward subtraction without regard to precision can erroneously yield a zero result, hence promoting a misconception that charge effects cancel beyond atomic bonding distances. To handle this issue, the Coulomb force equation must be rewritten as:

$$
F_{e 1 p 2}=\frac{k\left(-q_{e 1}\right)\left(+q_{p 2}\right)}{R^{2}-2 x_{e} R+x_{e}^{2}}
$$

By dividing the numerator by the denominator, a new force expression is obtained which can be handled without loss of precision:

$$
F_{e 1 p 2}=k\left(-q_{e 1}\right)\left(+q_{p 2}\right)\left[\frac{1}{R^{2}}+\frac{2 x_{e}}{R^{3}}+\frac{3 x_{e}^{2}}{R^{4}}+\frac{4 x_{e}^{3}}{R^{5}}+\ldots\right]
$$

The Monte Carlo numerical simulation process selects random positions for electrons around a spherical surface of radius $r$ for both the local and remote proton-electron pair, with $r$ representing the most probable proton-electron separation distance within an atom. The protons are positioned at known fixed locations separated by a distance $R$ along the $x$-axis at $x_{1}=0, y_{1}=0, z_{1}=0$ and $x_{2}=R, y_{2}=0, z_{2}=0$. Only $x$-axis components of force need be calculated based on the condition that movement of the electron likely places it at a mirror-image location on the opposite side of the $x$-axis sometime during the time period in question, 
resulting in cancellation of time-averaged force components normal to the $x$-axis. The $x$-axis represents any arbitrary direction outward from the local proton. The $x-y-z$ coordinates of electron positions with respect to a unity radius sphere are used to calculate the separation distances between local and remote. Let:

$$
\begin{aligned}
& d x=x_{2}-x_{1} \\
& d y=y_{2}-y_{1} \\
& d z=z_{2}-z_{1}
\end{aligned}
$$

The absolute distance $M$ between two sets of electron coordinate points is then given by:

$$
M^{2}=(R+r d x)^{2}+(r d y)^{2}+(r d z)^{2}
$$

The Coulomb force equation for any one of the four force components resolved to the $x$-axis component becomes (Appendix A):

$$
F=k q_{1} q_{2}\left[\frac{1}{R^{2}}-2 d x\left(\frac{r}{R^{3}}\right)+\left(3 d x^{2}-1.5 d y^{2}-1.5 d z^{2}\right)\left(\frac{r^{2}}{R^{4}}\right)-\ldots\right]
$$

The two attractive force components have a negative sign and the two repulsive force components have a positive sign. Summing the four force components for any set of local-remote points results in cancellation of the $1 / R^{2}$ terms. Likewise, with mirror-image points at negative $x$ as likely to occur as points at positive $x$, the $1 / R^{3}$ terms also cancel during averaging. Any terms for $1 / R^{5}, 1 / R^{6}$, and beyond are not significant with respect to the $1 / R^{4}$ term and are dropped. The result is:

$$
F=\frac{k q_{1} q_{2}}{R^{2}}\left[\left(3 d x^{2}-1.5 d y^{2}-1.5 d z^{2}\right)\left(\frac{r^{2}}{R^{2}}\right)\right]
$$

While the individual proton-electron charges experience forces that decrease with the inverse square of separation distance, the combining of a proton force with an electron force of opposite sign produces a sum that decreases with the inverse fourth power of separation distance. The force equation is still in the form of Coulomb's law but with an equivalent charge varying with the inverse square of separation. Let:

$$
H=3 d x^{2}-1.5 d y^{2}-1.5 d z^{2}
$$

Each of the four force components has a corresponding $H$ component, $H_{e l p 2}, H_{p l e 2}, H_{e l e 2}$, and $H_{p l p 2}$. From (10) $H$ is a quantity that adds to or subtracts from the basic attractive or repulsive force component, $1 / R^{2}$, depending upon the electron positions at any instant. Because $H$ is the only quantity that varies with different random electron positions over time, it is the only quantity that needs to be calculated by the Monte Carlo process. In that process, average values for $H_{e l p 2}, H_{p l e 2}$, and $H_{\text {ele } 2}$ are accumulated for repeated electron position selections. $H_{p l p 2}$ is always zero. The accumulation of all average $H$, representing the summing of the four force components, is then:

$$
H_{\text {avg }}=\text { average } H_{e l p 2}+\text { average } H_{p l e 2}-\text { average } H_{\text {ele2 }}
$$

A positive sign for $H_{\text {avg }}$ gives a negative sign for the net time-average force, meaning it is attractive in that case. $H$ is the single value, which if used to calculate net force between every local-remote proton-electron 
pair, provides the same average net force as accumulated for the actual random distribution of such pair forces throughout an increment of mass.

\section{SIMULATION RESULTS}

The Monte Carlo process requires making many runs simulating random electron positions for local and remote and then determining if the outcome for Havg produces a distinct, repeatable, non-random force pattern. To ensure no electron selection points on the spherical surface are computationally ignored during the computer simulation, the surface is pre-mapped with 41,258 equally spaced points each representing about one degree of arc length separation, and random selections are limited to the mapped points, each designated by a set of $x-y-z$ coordinates. This process provides a total of 1.7 billion possible random localremote electron pair positions. During the simulation each selection step randomly selects one of the 1.7 billion pair. Millions of random numbers need to be requested during any one simulation run, so it is necessary to eliminate the possibility of duplicating any sequence of random numbers, either during a run or from run to run. Sets of random numbers have been obtained from the internet site Random.org, a service built and operated at the School of Computer Science and Statistics at Trinity College, Dublin, Ireland, using atmospheric noise as its source.

Rewrite (12) for $H$ :

$$
H=3\left(x_{2}-x_{1}\right)^{2}-1.5\left(y_{2}-y_{1}\right)^{2}-1.5\left(z_{2}-z_{1}\right)^{2}
$$

Values for $x_{1}, x_{2}, y_{1}, y_{2}, z_{1}$, and $z_{2}$ vary from 0 to 1 . That means values for $H_{e l p 2}$ and $H_{p l e 2}$ can vary from -1.5 to +3.0 and $H_{\text {ele } 2}$ from -6.0 to +12.0 .

A pattern has emerged from simulations over a range of electron positions starting around 10,000 random selections and extending up through 10 million. The most consistent pattern occurs for 1 million or more pair selections. There is a distribution of $42 \%$ of the positions resulting in a positive $H$ and $58 \%$ a negative

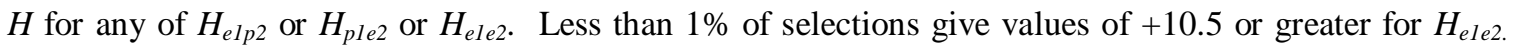
When these least probable values of $H_{\text {ele2 }}$ are excluded from the averaging, very distinct patterns emerge for $H_{\text {avg }}$. Each average $H$ is always negative with average $H_{\text {elez }}$ greater in magnitude compared to the sum of average $H_{\text {elp2 }}$ plus average $H_{p l e 2}$. This pattern means it is negative values of $H_{\text {ele2 }}$ that dominate the accumulation of total average value, making total $H_{\text {avg }}$ always a positive value and therefore, the total timeaveraged force always attractive. The physical interpretation of this pattern is that of all the possible locations for both local and remote electrons at any instant, there are sufficient occurrences of separation great enough to allow the time-averaged proton-electron attractive forces to always outweigh the combined electron-electron plus proton-proton repulsive forces, making the net residual time-averaged force always attractive. From thousands of simulation runs the most expected $H_{\text {avg }}$ consistently appears within the range of +0.005 to +0.12 with values around +0.013 being prominent .

\section{DERIVE NEWTON'S LAW OF GRAVITY}

Newton's law of gravity states the force between two masses is proportional to the product of the masses divided by the square of their separation distance:

$$
F=G \frac{m_{E} m_{R}}{R^{2}}
$$

where $m_{E}$ and $m_{R}$ are the mass quantities, $R$ is the separation distance, and $G$ is the proportionality constant or gravitational constant. To show equivalence with Newton's law, the Coulomb force equation should produce the same force on a mass at the earth's surface. However, it is not possible to calculate the 
Coulomb force for every individual charge pair in the earth given the immense number of charge pair, so an alternative approach is required. The calculation must start with forces that can be determined for a small increment of mass and build up a summation from there.

While it is convenient to develop the Coulomb force equations for the earth with respect to proton-electron charge pair within atoms, there is no physical significance to paring charges. The total Coulomb force between the earth and an object at the surface remains just the sum of forces between individual local and remote charges. This is the appropriate context in which to accumulate forces for the entire earth.

Newton proved that his law of gravity can be applied separately to each individual small increment of mass of a larger spherical mass with all the resulting forces for all increments summed to provide the total gravitational force of the larger mass as a whole. The individual forces reflect Newton's law using the mass of the incremental volume and the separation distance (resolved to the center-to-center axis) between the incremental volume and the remote point. The result of this process is that the gravitational force between two spherical masses of uniform density each can be equivalently calculated as if the entire mass of each was concentrated at the center point of the sphere and the separation distance used in the calculation is the center to center distance between the spheres [10]. This conclusion can also be verified via a numerical simulation.

Because Coulomb's law is mathematically equivalent to Newton's gravity law describing a force inversely proportional to the square of separation distance, the same principle applies to two spheres of uniform charge density each. The accumulation of all Coulomb charge forces over each sphere can be calculated as if all charge elements in the entire sphere were concentrated at the center point of the sphere and the separation distance used in the calculation is the center to center distance between the spheres. Coulomb's law can be applied in this manner for an increment of the earth versus a single remote charge pair at the earth's surface (Appendix C).

Consider all protons within a mass increment as one group of charge with an approximate uniform density. If all forces between each proton versus the remote proton are accumulated, the result is equivalent to making one calculation as if all protons for the increment are concentrated at the center of the increment. Likewise, all electrons as a group in the increment can be handled similarly. The forces for the two incremental charge groups can then be individually resolved to components along the $x$-axis connecting the earth center to the remote pair. Components normal to the $x$-axis cancel during time-averaging and are therefore not processed. The incremental charge groups along the $x$-axis can then be transferred to the earth center as equivalent charges with a magnitude which produces at the earth center the same Coulomb force with the remote. The result is that each incremental mass in the earth produces an equivalent proton charge and an equivalent electron charge at the earth center that can be combined into a single net charge with Coulomb components in the form of (11). The new net charge is then an equivalent charge representing the summing of multiple proton positive charge with multiple electron negative charge.

The equations for $H$ at the earth center remain identical to (12) and are calculated exactly as for a single local proton-electron pair. The force equations are multiplied times the count of all charge pair. If $N_{I}$ is the count of charge pair in the mass increment and $N_{R}$ is the count in the remote, then from (11) and (12) the total force effect at the earth center produced by a mass increment is:

$$
F_{I \ldots \text { origin }}=-\frac{k q_{p} q_{e}}{R^{2}} N_{I} N_{R} H_{\text {avg }}\left(\frac{r^{2}}{R^{2}}\right)
$$


Here count of charge pair for the increment equals mass of the increment $m_{I}$ divided by the mass per charge pair, which is approximated as the mass of a proton plus the mass of an electron plus the mass of a neutron, $m_{p e n}$. The same relation exists for the remote count.

$$
\begin{aligned}
& N_{I}=m_{I} / m_{p e n} \\
& N_{R}=m_{R} / m_{p e n}
\end{aligned}
$$

The Coulomb force equation can be verified by having it duplicate a quantity that can be measured experimentally, the acceleration of gravity at the earth's surface. Using Newton's law the acceleration of gravity at the earth's surface is described as the force per remote mass:

$$
a=\frac{F}{m_{R}}=G \frac{m_{E}}{R^{2}}
$$

where $a$ is the acceleration, $m_{E}$ is the earth mass, $m_{R}$ is the remote mass at the surface, and $G$ is the gravitational constant. The same relationship should hold with the Coulomb equation. A numerical analysis (Appendix C) run to accumulate incremental charge forces at the earth center verifies that the acceleration of gravity value is calculable via the Coulomb equation.

To complete derivation of Newton's law at the earth's surface, it is necessary to convert the total Coulomb force equation from charge to mass using the parameters listed in Table I. Let $M_{C}$ be the mass to charge ratio of a single proton-electron pair:

$$
\begin{gathered}
M_{C}=m_{p e n} / q \\
M_{C}=\left(2 \times 1.67 \times 10^{-27}+9.11 \times 10^{-31}\right) / 1.60 \times 10^{-19} \\
=2.09 \times 10^{-8}
\end{gathered}
$$

TABLE I. CONVERSION PARAMETERS

\begin{tabular}{ll} 
Coulomb's law constant, $k$ & $8.99 \times 10^{9} \mathrm{~N} \mathrm{~m}^{2} /$ coulomb $^{2}$ \\
proton charge, $q_{p}$ & $+1.60 \times 10^{-19}$ coulomb \\
electron charge, $-q_{e}$ & $-1.60 \times 10^{-19}$ coulomb \\
proton-electron average distance, $r$ & $1.0 \times 10^{-10} \mathrm{~m}$ \\
earth radius, $R_{E}$ & $6.38 \times 10^{6} \mathrm{~m}$ \\
gravitational constant, $G$ & $6.67 \times 10^{-11} \mathrm{~N} \mathrm{~m}^{2} / \mathrm{kg}^{2}$ \\
proton mass & $1.67 \times 10^{-27} \mathrm{~kg}$ \\
neutron mass & $1.67 \times 10^{-27} \mathrm{~kg}$ \\
electron mass & $9.11 \times 10^{-31} \mathrm{~kg}$ \\
earth mass & $5.98 \times 10^{24} \mathrm{~kg}$ \\
acceleration of gravity at earth surface & $9.80 \mathrm{~m} / \mathrm{s}^{2}$ \\
\hline
\end{tabular}

When the forces of all increments are summed at the earth center and all charge is summed at the earth center, the total Coulomb force equation becomes:

$$
\begin{gathered}
F_{\text {Coulomb }}=\sum F_{\text {I...origin }} \\
F_{\text {Coulomb }}=-\left[k H_{\text {avg }}\left(\frac{r}{R}\right)^{2}\left(\frac{1}{M_{C}}\right)^{2}\right] \frac{m_{E} m_{R}}{R^{2}}
\end{gathered}
$$




$$
F_{\text {Coulomb }}=-g \frac{m_{E} m_{R}}{R^{2}}
$$

Here $g$ is the quantity that needs to duplicate the gravitational constant. At the surface of the earth $g$ is:

$$
\begin{gathered}
g=\left(8.99 \times 10^{9}\right)\left(H_{\text {avg }}\right)\left(\frac{r}{6.38 \times 10^{6}}\right)^{2}\left(\frac{1}{2.09 \times 10^{-8}}\right)^{2} \\
g=5.056 \times 10^{11}\left(H_{\text {avg }}\right)\left(r^{2}\right)
\end{gathered}
$$

Two quantities remain uncertain in the force expression, $H_{\text {avg }}$ and the average proton-electron separation distance, $r$. The Monte Carlo simulation calculates probability distributions for $H$ and then accumulates a range of the most expected values for $H_{\text {avg }}$ by excluding the least probable values for $H$. A value for the proton-electron separation in any atom is not known with absolute certainty, but the most probable value is most often claimed by science to be on the order of $10^{-10}$ meters. A range for $r$ is also subject to the atoms of the earth being diverse but primarily oxygen, iron, and silicon, all with multiple electrons for which the proton-electron distance has uncertain values. Therefore a range of $r$ from $0.5-1.5 \times 10^{-10}$ has been selected to test the Coulomb force equation. If $r=1.0 \times 10^{-10}$, then $H_{\text {avg }}$ needs to be 0.0132 to give $g=6.67 \times 10^{-11}$, the gravitational constant. If $r=0.5 \times 10^{-10}$, then $H_{\text {avg }}$ needs to be 0.0528 , and if $r=1.5 \times$ $10^{-10}$, then $H_{\text {avg }}$ needs to be 0.00586 . All of these values for $H_{\text {avg }}$ lie within the range of +0.005 to +0.12 identified by the simulation runs as the most expected $H_{\text {avg }}$. The resulting range of $g$ places it very near the value of the gravitational constant supporting a conclusion that the expression for $F_{\text {Coulomb }}$ is Newton's law and that gravity is therefore an electromagnetic force and the electric charge force of protons and electrons is the source of the gravitational force.

\section{CONCLUSION}

There should now be an understanding that charge effects extend well beyond the confines of the immediate atom despite zero net charge of the atom and its surrounding atoms. Whereas electrostatics is the interaction of stationary charges and magnetism is the interaction of moving charges, gravity is the interaction of charge pair. The evidence provided herein showing that gravity is an electromagnetic force and not a unique force of its own provides a significant contribution toward reconciling the long sought link between gravity and electricity.

\section{APPENDIX A \\ FORCE EQUATION DERIVATION}

During the computerized Monte Carlo numerical process, when electron positions are randomly selected around a spherical surface for both local and remote locations, their $x-y-z$ coordinates are used to calculate the separation distances between local and remote (Fig. A1). Position 1 is local and position 2 is remote. The protons are $p_{1}$ and $p_{2}$ and the electrons are $e_{1}$ and $e_{2} . \quad R$ is the proton-proton separation distance. $M$ is the electron-electron separation distance. Let:

$$
\begin{aligned}
& d x=x_{2}-x_{1} \\
& d y=y_{2}-y_{1} \\
& d z=z_{2}-z_{1}
\end{aligned}
$$




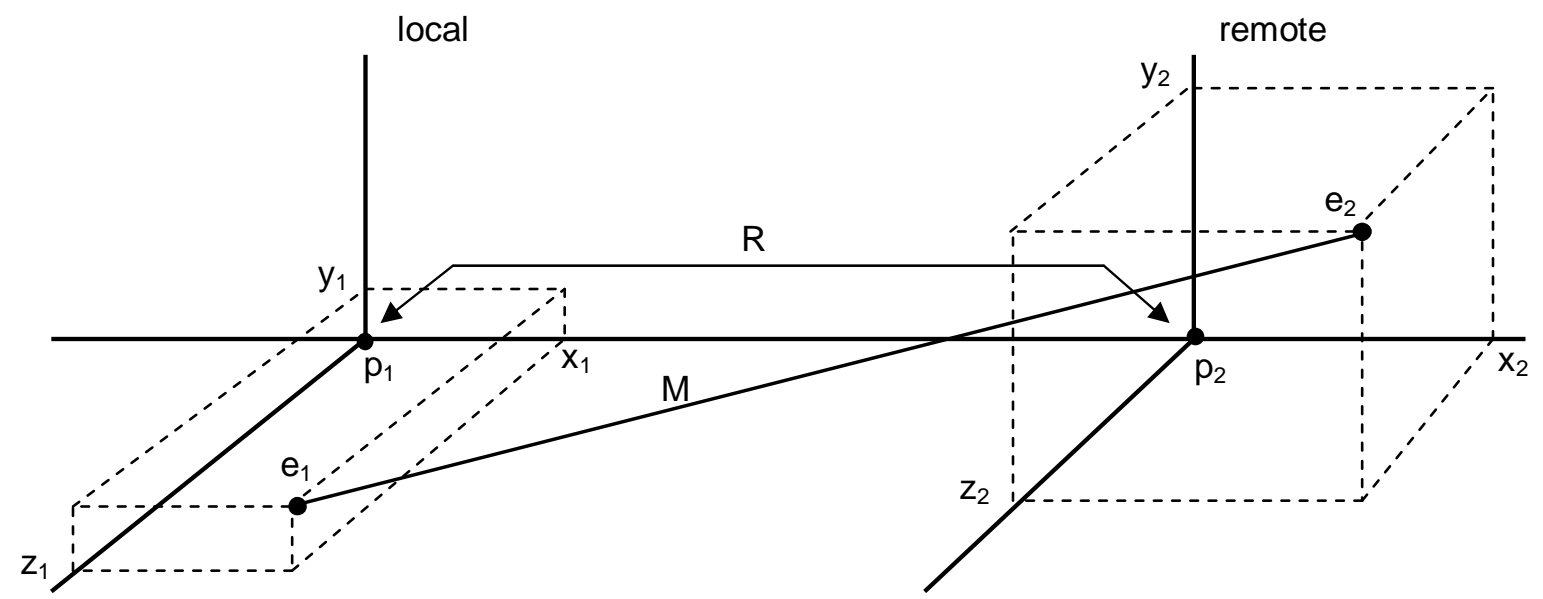

Fig A1. Calculating Distance M Between Local and Remote Electrons

Since these are coordinates for a unity radius sphere, each must be multiplied by the actual sphere radius $r$, the average proton-electron separation distance. The absolute distance $M$ between two sets of electron coordinate points is then given by:

$M^{2}=(R+r d x)^{2}+(r d y)^{2}+(r d z)^{2}$

The resulting electron-electron Coulomb force equation, resolved to its $x$-axis component, is:

$F=\frac{k q_{1} q_{2}}{M^{2}}\left[\frac{R+r d x}{M}\right]$

This expression needs to be resolved into the form:

$F=k q_{1} q_{2}\left[\frac{1}{R^{2}}+\frac{2 x_{e}}{R^{3}}+\frac{3 x_{e}^{2}}{R^{4}}+\frac{4 x_{e}^{3}}{R^{5}}+\ldots\right]$

To accomplish this it is first expressed as a single fraction:

$$
\begin{aligned}
& F=k q_{1} q_{2}\left[\frac{1}{(R+r d x)^{2}+(r d y)^{2}+(r d z)^{2}}\right]\left[\frac{\sqrt{(R+r d x)^{2}}}{\sqrt{(R+r d x)^{2}+(r d y)^{2}+(r d z)^{2}}}\right] \\
& =k q_{1} q_{2}\left[\sqrt{\left.\frac{1}{\left[(R+r d x)^{2}+(r d y)^{2}+(r d z)^{2}\right]^{2}}\right]}\left[\sqrt{\frac{(R+d x)^{2}}{(R+r d x)^{2}+(r d y)^{2}+(r d z)^{2}}}\right]\right. \\
& =k q_{1} q_{2}\left[\sqrt{\frac{(R+r d x)^{2}}{\left[(R+r d x)^{2}+(r d y)^{2}+(r d z)^{2}\right]^{3}}}\right] \\
& =k q_{1} q_{2}\left[\sqrt{\frac{R^{2}+2 r d x R+(r d x)^{2}}{\left[R^{2}+2 r d x R+(r d x)^{2}+(r d y)^{2}+(r d z)^{2}\right]^{3}}}\right]
\end{aligned}
$$

After expanding the polynomial in the denominator and then dividing the numerator by the denominator, the result is: 


$$
F=k q_{1} q_{2}\left[\frac{1}{R^{2}} \sqrt{1-\frac{4 r d x}{R}+\frac{10(r d x)^{2}-3(r d y)^{2}-3(r d z)^{2}}{R^{2}}-\ldots}\right]
$$

Then, where $\Delta a<<1.0$, the following can be used:

$$
\sqrt{1+\Delta a}=1+\frac{\Delta a}{2}-\frac{\Delta a^{2}}{8}+\frac{\Delta a^{3}}{16}-\ldots
$$

In this case:

$$
\begin{aligned}
& \Delta a=-\frac{4 r d x}{R}+\frac{10(r d x)^{2}}{R^{2}}-\frac{3(r d y)^{2}}{R^{2}}-\frac{3(r d z)^{2}}{R^{2}}-\ldots \\
& \frac{\Delta a^{2}}{8}=\frac{1}{8}\left[\frac{16(r d x)^{2}}{R^{2}}-\frac{8 r d x\left[10(r d x)^{2}-3(r d y)^{2}-3(r d z)^{2}\right]}{R^{3}}+\frac{\left[10(r d x)^{2}-3(r d y)^{2}-3(r d z)^{2}\right]^{2}}{R^{4}}+\ldots\right] \\
& =\frac{2(r d x)^{2}}{R^{2}}+\ldots
\end{aligned}
$$

Therefore:

$$
\begin{aligned}
& \sqrt{1+\Delta a}=1-\frac{2 r d x}{R}+\frac{\left[5(r d x)^{2}-1.5(r d y)^{2}-1.5(r d z)^{2}\right]}{R^{2}}-\frac{2(r d x)^{2}}{R^{2}}+\ldots \\
& F=k q_{1} q_{2} \frac{1}{R^{2}}\left[1-\frac{2 r d x}{R}+\frac{\left[3(r d x)^{2}-1.5(r d y)^{2}-1.5(r d z)^{2}\right]}{R^{2}}-\ldots\right] \\
& F=k q_{1} q_{2}\left[\frac{1}{R^{2}}-2 d x\left(\frac{r}{R^{3}}\right)+\left(3 d x^{2}-1.5 d y^{2}-1.5 d z^{2}\right)\left(\frac{r^{2}}{R^{4}}\right)-\ldots\right]
\end{aligned}
$$

The four force components for two proton-electron pair become (where $q_{e}$ and $q_{p}$ are represented as the magnitudes of proton and electron charge and therefore $\left.q_{e}=q_{p}\right)$ :

$$
\begin{aligned}
& F_{e 1 p 2}=k\left(-q_{e}\right) q_{p}\left[\frac{1}{R^{2}}-2\left(-x_{1}\right)\left(\frac{r}{R^{3}}\right)+\left[3\left(-x_{1}\right)^{2}-1.5\left(-y_{1}\right)^{2}-1.5\left(-z_{1}\right)^{2}\right]\left(\frac{r^{2}}{R^{4}}\right)-\ldots\right] \\
& F_{p l e 2}=k q_{p}\left(-q_{e}\right)\left[\frac{1}{R^{2}}-2\left(x_{2}\right)\left(\frac{r}{R^{3}}\right)+\left[3\left(x_{2}\right)^{2}-1.5\left(y_{2}\right)^{2}-1.5\left(z_{2}\right)^{2}\right]\left(\frac{r^{2}}{R^{4}}\right)-\ldots\right] \\
& F_{e l e 2}=k\left(-q_{e}\right)\left(-q_{e}\right)\left[\frac{1}{R^{2}}-2\left(x_{2}-x_{1}\right)\left(\frac{r}{R^{3}}\right)+\left[3\left(x_{2}-x_{1}\right)^{2}-1.5\left(y_{2}-y_{1}\right)^{2}-1.5\left(z_{2}-z_{1}\right)^{2}\right]\left(\frac{r^{2}}{R^{4}}\right)-\ldots\right] \\
& F_{p 1 p 2}=k q_{p} q_{p}\left[\frac{1}{R^{2}}\right]
\end{aligned}
$$

For each component a value of $H$ is established as:

$$
H=3\left(x_{2}-x_{1}\right)^{2}-1.5\left(y_{2}-y_{1}\right)^{2}-1.5\left(z_{2}-z_{1}\right)^{2}
$$


Summing the four force components results in cancellation of the $1 / R^{2}, 1 / R^{3}$, and $1 / R^{5}$ terms. Terms beyond are not significant and are dropped. The relevant time-averaged values become:

$$
\begin{aligned}
& \left(F_{e 1 p 2}\right)_{a v g}=-k q_{e} q_{p}\left(H_{e 1 p 2}\right)_{a v g}\left[\frac{r^{2}}{R^{4}}\right] \\
& \left(F_{p 1 e 2}\right)_{a v g}=-k q_{p} q_{e}\left(H_{p 1 e 2}\right)_{a v g}\left[\frac{r^{2}}{R^{4}}\right] \\
& \left(F_{\text {ele2 }}\right)_{\text {avg }}=+k q_{e} q_{e}\left(H_{e l e 2}\right)_{\text {avg }}\left[\frac{r^{2}}{R^{4}}\right] \\
& H_{p 1 p 2}=0 \\
& \left(F_{\text {total }}\right)_{\text {avg }}=\left(F_{\text {e1 } p 2}\right)_{\text {avg }}+\left(F_{p 1 e 2}\right)_{a v g}+\left(F_{\text {ele } 2}\right)_{\text {avg }} \\
& \left(F_{\text {total }}\right)_{\text {avg }}=-k q q\left[\left(H_{e 1 p 2}\right)_{\text {avg }}+\left(H_{p 1 e 2}\right)_{\text {avg }}-\left(H_{\text {ele2 }}\right)_{\text {avg }}\right]\left[\frac{r^{2}}{R^{4}}\right] \quad \text { where } q=q_{e}=q_{p} \\
& \left(F_{\text {total }}\right)_{\text {avg }}=-k q q H_{\text {avg }}\left[\frac{r^{2}}{R^{4}}\right] \\
& H_{a v g}=\left(H_{e l p 2}\right)_{a v g}+\left(H_{p l e 2}\right)_{a v g}-\left(H_{e l e 2}\right)_{a v g} \\
& H_{\text {avg }}=\text { average } H_{e l p 2}+\text { average } H_{p l e 2} \text { - average } H_{\text {ele } 2}
\end{aligned}
$$

\section{APPENDIX B \\ MONTE CARLO SIMULATION SOFTWARE}

The computerized method consists of:

(1) Simulate $F_{e l p 2}$ and $F_{p l e 2}$ proton-electron forces by calculating $H_{e l p 2}$ and $H_{p l e 2}$ for two random electron coordinate points, one local and one remote, and accumulate two averages for all such calculations.

(2) Simulate $F_{\text {elez }}$ electron-electron forces by using the same two selected random electron coordinate points. Calculate $H_{\text {ele2 }}$ for the pair and then accumulate an average for all $H_{\text {ele2 }}$.

(3) Because $F_{p l p 2}$ proton-proton forces are represented by only a $1 / R^{2}$ term, $H_{p l p 2}$ is always zero.

(4) Repeat this process many times with different random selections for electron locations and accumulate running averages for each $H$.

(5) Sum the three resulting average $H$ from above.

(6) Interpret signs as follows: Both equations for $F_{e l p 2}$ and $F_{p l e 2}$ should have negative signs since each is an attractive force while the equation for $F_{\text {ele2 }}$ should have a positive sign as a repulsive force. A positive sign for average $H_{e l p 2}$ or average $H_{p l e 2}$ contributes a negative sign to $F_{e l p 2}$ and $F_{p l e 2}$, meaning it is attractive in that case. A positive sign for $H_{e l e z}$ contributes a positive sign to $F_{e l e z}$, meaning it is repulsive.

The accumulation of total average $H$ is:

$$
H_{\text {avg }}=\text { average } H_{e l p 2}+\text { average } H_{p l e 2}-\text { average } H_{\text {ele2 }}
$$

Flow of the software processes is: 
- Pre-map a sphere surface with equally spaced points to be selected for random electron positions and save in an array the $x, y, z$ coordinates for 41,258 total entries.

- Initialize the random number process:

- Create array of 20,000 random integers ranging from 1 to 99,999 obtained from the internet site, Random.org.

- Add leading zeroes so all integers are 5 digits in length and save the resulting 100,000 digits in another array from which any single digit can be eventually selected.

- Set up 16 probability ranges into which count of values of $H$ can be accumulated for each range, i.e., slot count of $H$ versus magnitude of $H$, including both negative and positive magnitudes.

- Run the Monte Carlo simulation by looping through a random selection and calculation process a specified number of times, typically one million:

- Start to calculate a value for $H_{e l p 2}$ by selecting a random electron position on the sphere surface. Randomly select 5 digits from the 100,000-entry character array to create a random decimal 0.xxxxx and use this decimal to identify one of the entries in the 41,258-entry array of $x, y, z$ coordinate points.

- The second position for $H_{e l p 2}$ is the proton position at $x=0, y=0, z=0$.

- Calculate the value for $H_{\text {elp2 }}$ and accumulate magnitude and count in the appropriate probability range.

- Repeat for $H_{p l e 2}$, by selecting 5 digits to identify another one of the 41,258-entry array entries.

- Perform a calculation for $H_{\text {elez }}$ by using the two electron positions just selected, assuming one is local and the other is remote. Accumulate magnitude and count in the appropriate probability range.

- Repeat for the specified number of times.

- After completing these calculations the specified number of times, say one million, three sets of accumulated count by positive magnitude and three sets of accumulated count by negative magnitude are available for $H_{e l p 2}, H_{p l e 2}$, and $H_{e l e 2}$ for each of the 16 probability ranges.

TABLE II. SAMPLE RESULTS

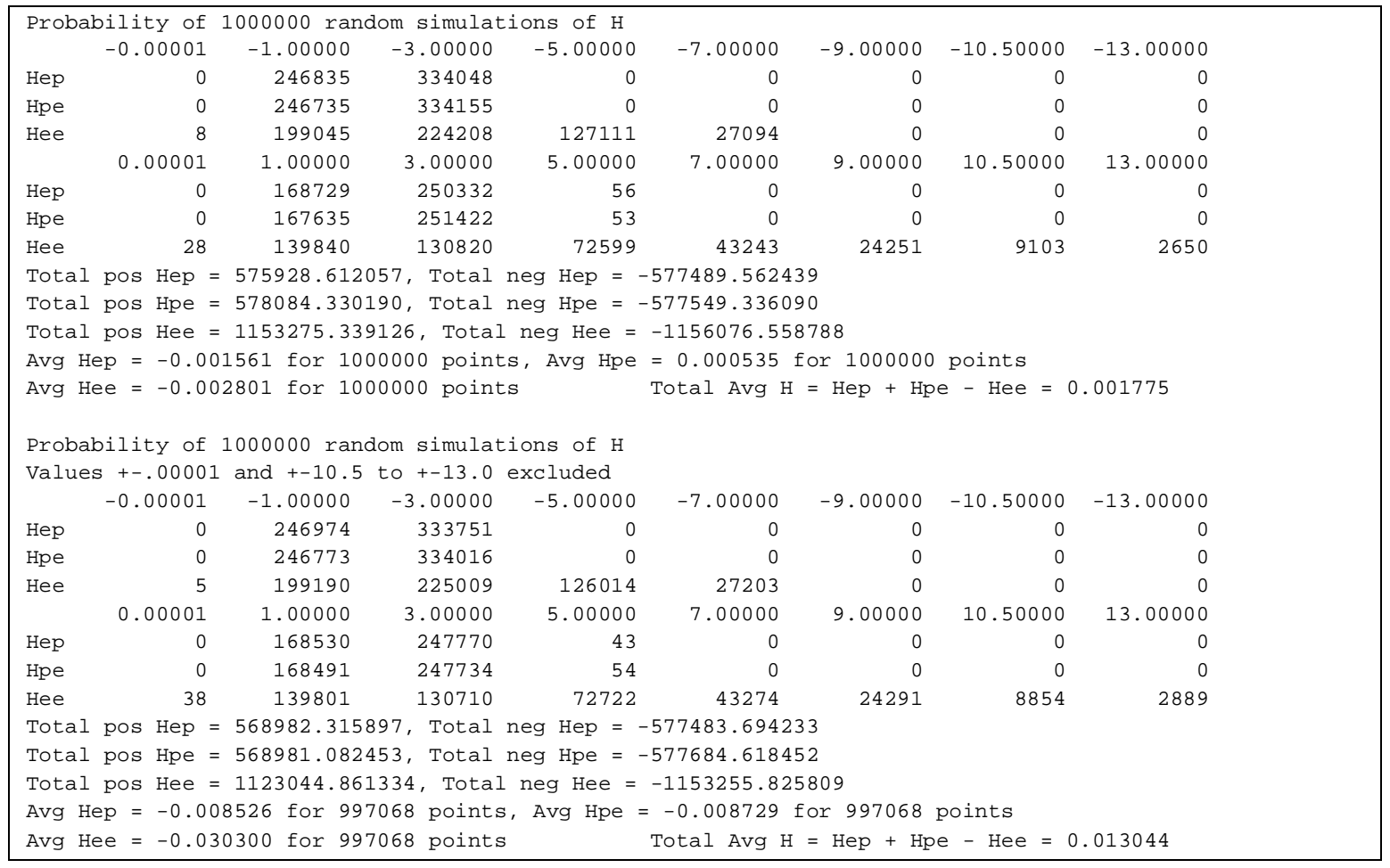


- Using the accumulated magnitudes and counts, calculate $H_{\text {avg }}$ per (A-1).

- Print table of results (samples: Table II). The value for any entry in the table represents a count of the number of occurrences of a value of $H$ within a range between the column heading and the column heading to the left, including the number in the column heading to the left. For example, column heading +5.0 represents count of occurrences of $H$ greater or equal +3.0 and less than +5.0 .

\section{APPENDIX C}

ACCUMULATE INCREMENTAL CHARGE OF THE EARTH

To verify the Coulomb force equation, a computerized numerical process can be run to accumulate charge for incremental volumes of mass of the earth and reflect equivalent charge to the center of the earth such that the sum of forces of the equivalent charge at the earth center with respect to a remote location equals

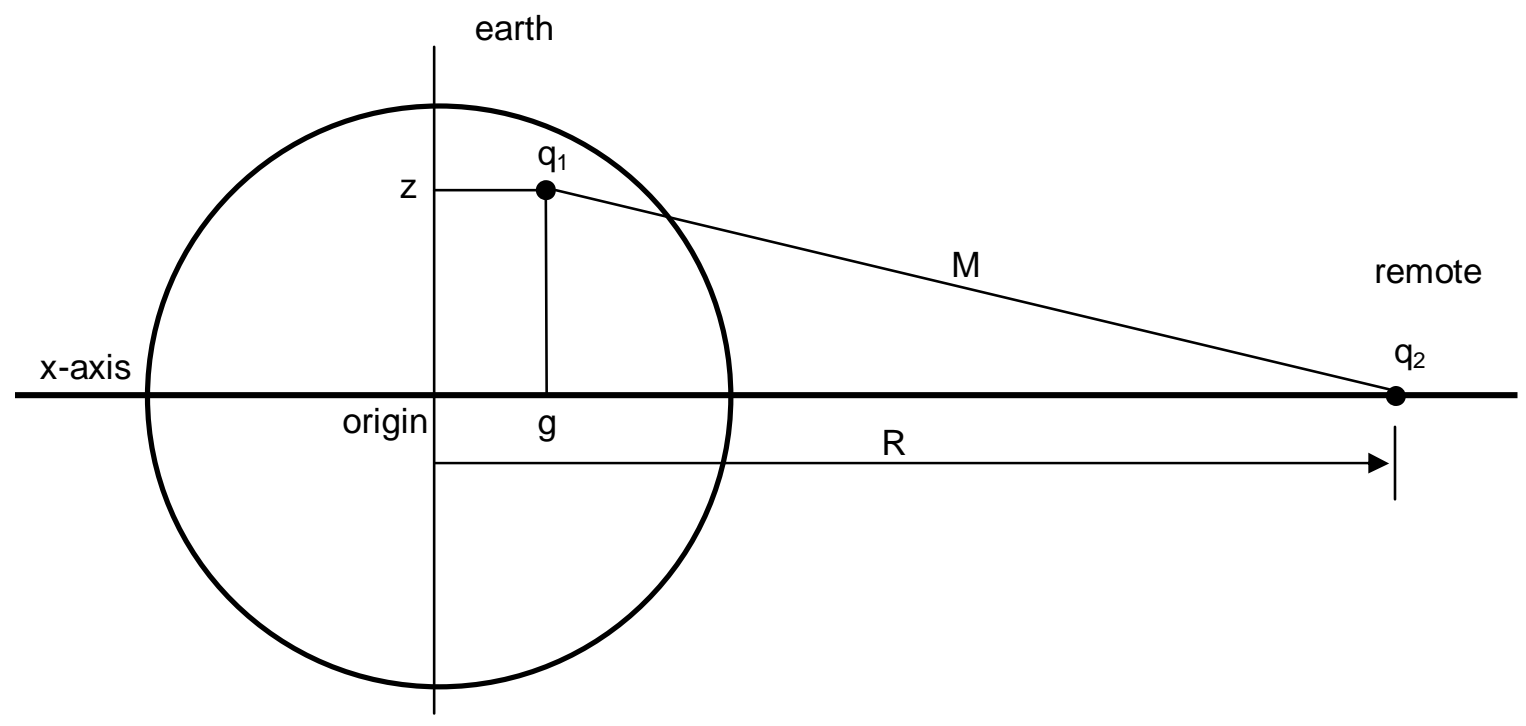

Fig A2. Earth in $x-y-z$ Coordinates vs. Point Mass

the sum of charge forces of the original increments with respect to the remote location. Then the positive and negative charge, each accumulated separately at the earth center, can be combined to give the Coulomb force equation which can be compared to Newton's law at the earth center. Let position 1 be a location within the earth and position 2 the remote location (Fig. A2).

Start with the earth as a sphere of uniform proton density and a proton point charge as the remote charge. Divide the earth into incremental volumes and concentrate all protons within each increment at the center of the increment on the basis that the sum of Coulomb forces for all protons within the increment with respect to the remote charge equals the single force calculated as if all protons are concentrated at the center of the increment. This basis will be verified by the numerical process. Total Coulomb proton force for any increment is:

$\Delta F_{I}=\frac{k \Delta N_{I} N_{R} q_{1} q_{2}}{M^{2}}$

$\Delta N_{I}=\frac{m_{E} \Delta V}{m_{p e n} V_{E}} \quad N_{R}=\frac{m_{R}}{m_{p e n}}$ 
$M^{2}=(R-g)^{2}+z^{2}$

where $\Delta N_{I}$ is the total proton count in the increment, $N_{R}$ is the total remote proton count $(=1), \quad q 1$ and $q_{2}$ are the charge of a proton, $R$ is the distance from the earth center to the remote charge, $M$ is the distance from the increment to the remote charge, $\Delta V$ is the volume of the increment, $V_{E}$ is the earth volume, $m_{E}$ is the earth mass, $m_{R}$ is the remote mass, and $m_{p e n}$ is the mass of a single proton-electron-neutron combination.

Resolve each increment force to an $x$-axis force where the $x$-axis is the straight line connecting the earth center to the remote point. Only $x$-axis forces are considered since $y$-axis and $z$-axis forces cancel during summation.

$\Delta F_{I x-a x i s}=\Delta F_{I} \cos \Phi=\Delta F_{I}\left(\frac{R-g}{M}\right)$

where $\Phi$ is the angle between $M$ and the $x$-axis, $g$ is the $x$ coordinate of the increment, and $g_{x}$ is the location on the $x$-axis where the force $\Delta F_{I x \text {-axis }}$ occurs using the original increment charge $\left(g_{x}\right.$ not equal to $\left.g\right)$. Then:

$\Delta F_{I x-a x i s}=\frac{k \Delta N_{I} N_{R} q_{1} q_{2}}{\left(R-g_{x}\right)^{2}}$

The above two equations can be solved for $\left(R-g_{x}\right)^{2}$ :

$\left(R-g_{x}\right)^{2}=\frac{M^{2} M}{R-g}$

The incremental proton charge located on the $x$-axis can then be transferred to the earth center (origin) as a new equivalent charge to give the same force with respect to the remote as the incremental $x$-axis force. This transfer is done by multiplying the charge times a transfer factor:

$\operatorname{Chrg}_{\text {origin }}=\operatorname{Chrg}_{x-a x i s} \frac{R^{2}}{\left(R-g_{x}\right)^{2}}$

$\operatorname{Chrg}_{\text {origin .. proton }}=\frac{m_{E}}{m_{\text {pen }}} \frac{m_{R}}{m_{p e n}} q_{1} q_{2} \frac{\Delta V}{V_{E}} \frac{R^{2}}{\left(R-g_{x}\right)^{2}}$

When all equivalent incremental charges are summed at the earth center, the force they produce with the remote force at a distance $R$ from the earth center represents the total force of all protons in the earth taken together. This relation can be verified by running a numerical simulation of the following summation. It can be concluded that the sum of all individual incremental charges with the remote is equivalent to making one calculation with all incremental charges summed as one charge at the earth center.

$F_{\text {origin ...proton }}=k\left[\sum\right.$ Chrg $\left._{\text {origin ...proton }}\right] \frac{1}{R^{2}}$ 


$$
F_{\text {origin } \ldots \text { proton }}=k\left[\sum\left(\frac{1}{V_{E}} \frac{m_{E}}{m_{p e n}} \frac{m_{R}}{m_{p e n}} q_{1} q_{2} \frac{R^{2}}{\left(R-g_{x}\right)^{2}}\right) \Delta V\right] \frac{1}{R^{2}}
$$

If the right side of the expression is replaced with its equivalent:

$\frac{R^{2}}{\left(R-g_{x}\right)^{2}}=\left(\frac{R-g}{M^{2} M}\right) R^{2}$

then the total force at the origin becomes:

$$
\begin{aligned}
F_{\text {originn... proton }}= & k \sum \frac{1}{V_{E}} \frac{m_{E}}{m_{p e n}} \frac{m_{R}}{m_{p e n}} q_{1} q_{2}\left(\frac{R-g}{M^{2} M}\right) \Delta V \\
& =\sum \frac{k \Delta N_{I} N_{R} q_{1} q_{2}}{M^{2}}\left(\frac{R-g}{M}\right)
\end{aligned}
$$

This force is the summation of $x$-axis components of the original incremental charge forces. It also proves that transferring all incremental charge to the earth center as one equivalent charge produces the same force with the remote as summing the forces of the original incremental charges.

The next step is to process all electrons in the earth similarly as a second group of charge with a uniform density. The same equations will result but with the opposite sign. When both sets of charge equations are combined at the earth center the forces for these two equivalent charges then produce the four Coulomb force components $F_{\text {elp } 2}, F_{p l e 2}, F_{\text {ele2 }}$, and $F_{p l p 2}$, which when summed have an $H_{\text {avg }}$ component.

Let the mass to charge ratio be:

$M_{C}=m_{p e n} / q_{1}=m_{p e n} / q_{2}$

Then:

$$
\begin{aligned}
F_{\text {total }} & =F_{e 1 p 2}+F_{p 1 e 2}+F_{e l e 2}+F_{p 1 p 2} \\
& =\sum \frac{1}{V_{E}}\left(\frac{k H_{\text {avg }} r^{2}}{M_{C}^{2}}\right) m_{E} m_{R}\left(\frac{R-g}{M^{2} M}\right) \frac{\Delta V}{R^{4}}
\end{aligned}
$$

When $R$ is set equal to the earth radius, the above can be expressed as the acceleration of gravity at the earth surface:

$$
\text { accel }=\frac{F_{\text {total }}}{m_{R}}=\left(\frac{3}{4 \Pi R_{E}^{3}}\right)\left(\frac{k H_{\text {avg }} r^{2}}{M_{C}^{2}}\right) \frac{m_{E}}{R^{4}}\left[\sum \frac{(1-g / R) \Delta V}{\left[1-2 g / R+g^{2} / R^{2}+z^{2} / R^{2}\right]^{3 / 2}}\right]
$$

If Havg $=0.013$ and $r=1.0 \times 10^{-10}$, the computer simulation of the above summation at $R=R_{E}$ gives an acceleration value of 9.8 , the same acceleration calculated via Newton's law. The acceleration is the appropriate parameter to match since it can be verified by experiment. 


\section{ACKNOWLEDGMENT}

The author thanks the staff at the School of Computer Science and Statistics, Trinity College, Dublin, Ireland, and Randomness and Integrity Services Ltd., Dublin, Ireland, for contributing random number sets supplied over the internet site, Random.org.

\section{REFERENCES}

1. Newton I. Cohen I, Whitman A translators. The principia: mathematical principles of natural philosophy. Berkeley: Univ. CA Press; 1999. Book 3 pp. 791- 938.

2. Einstein A. The foundation of the general theory of relativity. Annalen der Physik 1916; 49.

3. Einstein A. Relativity: The special \& the general theory 100th anniversary edition. Princeton: Princeton University Press; 2015.

4. Clark RW. Einstein the life and times. New York: HarperCollins Publishers; 1971. pp. 492-495.

5. Feynman RP, Morinigo FB, Wagner WG. Hatfield B., editor. Feynman lectures on gravitation. Boulder: Westview Press; 2003. pp. 2, 15.

6. Caillon JC. A possible unification of Newton's and Coulomb's forces. Physics Letters A 2018; 386; 46; pp. 3307-3312.

7. Coulomb CA. Premier mémoire sur l'électricité et le magnétisme. Histoire de l'Académie Royale des Sciences 1785; pp. 569-577.

8. Coulomb CA. Second mémoire sur l' électricité et le magnétisme. Histoire de l'Académie Royale des Sciences 1785; pp. 578-611.

9. Metropolis N, Ulam S. The Monte Carlo method. Journal American Statistical Assoc. 1949; 44; pp. 335-341.

10. Newton I. Cohen I., Whitman A. translators. The principia: mathematical principles of natural philosophy. Berkeley: Univ. CA Press; 1999. Book 1 pp. 431-629. 